\title{
An efficient design of 45-nm CMOS low-noise charge sensitive amplifier for wireless receivers
}

\author{
Islam T. Almalkawi ${ }^{1}$, Ashraf H. Al-Bqerat ${ }^{2}$, Awni Itradat ${ }^{1}$, Jamal N. Al-Karaki $^{3}$ \\ ${ }^{1}$ Computer Engineering Department (CPE), Faculty of Engineering, Hashemite University, Zarqa, Jordan \\ ${ }^{2}$ Department of Electrical and Computer Engineering (ECE), Concordia University, Montreal, Canada \\ ${ }^{3}$ Information Security Engineering Technology Department, Abu Dhabi Polytechnic, Abu Dhabi, United Arab Emirates
}

\begin{abstract}
Article Info
Article history:

Received Feb 23, 2021

Revised Jul 19, 2021

Accepted Aug 16, 2021

\section{Keywords:}

Charging capacitance

Complementary metal oxide

semiconductor

Constant voltage scaling

Equivalent noise charge

Front-end circuits

Preamplifier

ABSTRACT

Amplifiers are widely used in signal receiving circuits, such as antennas, medical imaging, wireless devices and many other applications. However, one of the most challenging problems when building an amplifier circuit is the noise, since it affects the quality of the intended received signal in most wireless applications. Therefore, a preamplifier is usually placed close to the main sensor to reduce the effects of interferences and to amplify the received signal without degrading the signal-to-noise ratio. Although different designs have been optimized and tested in the literature, all of them are using larger than $100 \mathrm{~nm}$ technologies which have led to a modest performance in terms of equivalent noise charge (ENC), gain, power consumption, and response time. In contrast, we consider in this paper a new amplifier design technology trend and move towards sub $100 \mathrm{~nm}$ to enhance its performance. In this work, we use a pre-well-known design of a preamplifier circuit and rebuild it using $45 \mathrm{~nm}$ CMOS technology, which is made for the first time in such circuits. Performance evaluation shows that our proposed scaling technology, compared with other scaling technology, extremely reduces ENC of the circuit by more than $95 \%$. The noise spectral density and time resolution are also reduced by $25 \%$ and $95 \%$ respectively. In addition, power consumption is decreased due to the reduced channel length by $90 \%$. As a result, all of those enhancements make our proposed circuit more suitable for medical and wireless devices.
\end{abstract}

This is an open access article under the $\underline{C C B Y-S A}$ license.

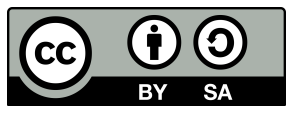

\author{
Corresponding Author: \\ Islam T. Almalkawi \\ Computer Engineering Department (CPE), Faculty of Engineering, Hashemite University \\ Damascus Hwy, Zarqa, Jordan \\ Email eslam.malkawi@hu.edu.jo
}

\section{INTRODUCTION}

Massive researches have been healed throughout decades in many wireless applications, medical imaging, and radiation detectors front-end circuits. As received signal has low amplitude and low Signalto-noise ratio (SNR). It should be treated and manipulated by a circuit that takes the desired signal and wipes out or filters other noise signals, which is mainly done by a preamplifier. This preamplifier is an operational amplifier (OpAmp) used in amplifier integrator mode by using a feedback capacitance. The integrator mode of operation enables OpAmps to integrate weak charge pulses into voltage pulses. A preamplifier is used to enhance the SNR by converting the incoming charge into a usable voltage pulse signal in order to process it in later stages using the remaining components of the readout system. Because of that, this type of amplifier is called "charge amplifier" [1]-[4]. The integration mode amplifiers have very high input impedance, while the 
output stage is a low-impedance buffer to drive a detected signal to the remaining components of the system. Figure 1 illustrates the basic charge sensitive amplifier (CSA) [1], [2], [5], [6].

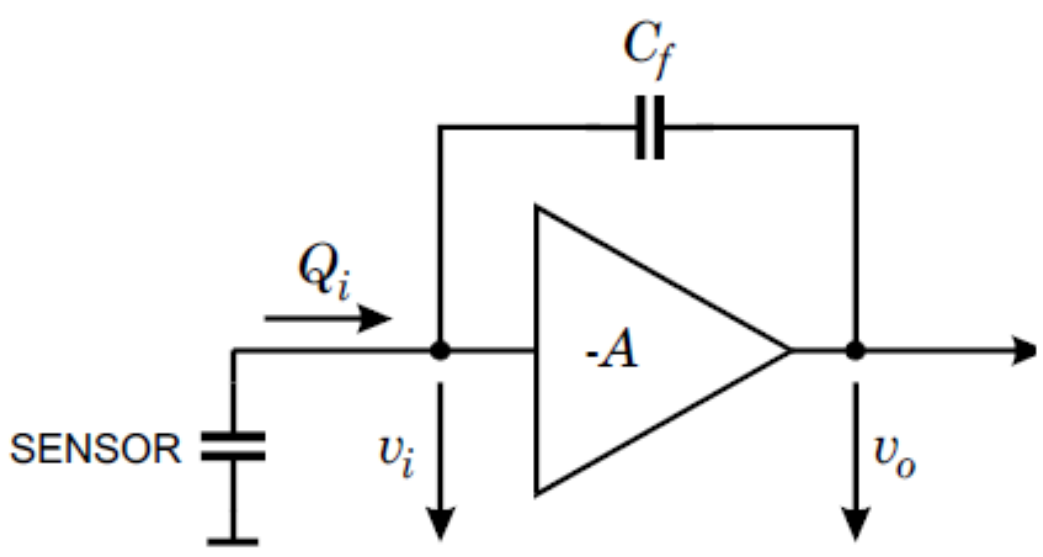

Basic configuration of a charge-sensitive amplifier

Figure 1. Basic charge sensitive amplifier

When looking at the transistor level, complementary metal-oxide-semiconductor (CMOS)-based technology is the dominant technology for CSA circuits and even for any other low-power amplifier application. Low noise field effect transistor (FET) comes as a first stage in the charge amplifiers [1]-[4]. The charge amplifier's open-loop gain is set sufficiently high so that the amplification is not affected by the detector capacitance. On the other hand, the input metal oxide semiconductor field effect transistor (MOSFET) of the preamplifier is designed and optimized to be the dominant noise source of the entire system. Many research papers made comparisons on the usage of junction field effect transistors (JFETs), bipolar junction transistors (BJTs), gallium arsenid metal semiconductor field effect transistor (GaAs MESFET), and CMOS [6] in terms of cost, SNR, and power consumption. In these comparisons, CMOS proved its advantages over other transistors. MOSFET has a high transconductance [7], [8] that generates smaller noise; this is useful for high-resolution images. Also, its low power consumption makes it better for high-dense detectors applications. As an example of the importance of low power consumption, a high-resolution positron emission tomography (PET) scanner system, manufactured by lutetium oxyorthosilicate (LSO) crystals coupled to avalanche photodiode (APD) arrays, uses up to $55 \mathrm{~K}$ preamplifiers [6]. If we assume that a single CSA requires $1 \mathrm{~mA}$ current, then the PET scanner requires a total of $55 \mathrm{~A}$. Thus, this example shows us that developing CSA is a very crucial issue regarding its power consumption [3], [4]. As a result, all state of art papers agree on CMOS and only CMOS to use in designing preamplifiers [9]-[13], but they still negotiate on which CMOS topology is more efficient.

Since the 90s of the last century, CMOS-based CSA circuits became technology dominant till now. From that time, all designers benefited from CMOS deep scaling down approach. Starting from 2 m CMOS [3], [4] down to $0.8 \mathrm{~m} \mathrm{CMOS}$ in early 2000 [2], reaching now values of $45 \mathrm{~nm}$ nowadays. This trend accompanying designs proved its advantages for high resolution and small scale applications [14], [15]. As CSA is used in high power consuming applications, it needs to reduce the power it dissipates. In our work, we have concentrated on overcoming ENC and power consumption issues. These two critical issues are the most important characteristics that a designer should take into consideration for Femto-coulomb signals receiving applications like PET scanners, wireless sensor networks, and IoT applications [16]-[18].

In this paper, an efficient design of a CMOS regulated cascode topology preamplifier is presented. In this proposal, a well-known topology is used in such circuits but with $45 \mathrm{~nm}$ scaling technology. Simulation results have shown that the performance of the proposed design has been improved significantly: a microwatt CSA with lower e-rms ENC, reduced power spectral density, reduced timing resolution, and faster response time compared with other scaling technologies. Those enhancements make our circuit ideal for wireless applications where ultra-low power weak signal receivers and higher resolution images are used. 
The rest of the paper is organized as follows: Section 2 reviews recent work in cascode topologies CSA circuits used in PET applications. Our system design preliminaries are described in section 3. Section 4 discusses scaling techniques used in our proposed circuit. Section 5 introduces our modified telescopic cascode topology CSA. Section 6 shows the simulation results of our proposed design compared to other related works, and section 7 ends the paper with conclusions and future work.

\section{RELATED WORK}

Many conducted research through the last decades has led to many advances in the medical imaging field. All design trends head towards reducing channel length in FET to enhance image quality [19]-[22] and thus it is going toward more FET shrinking, this trend proves that it is very powerful and efficient in terms of reducing ENC ratio and improving image quality.

Schmitt et al. [6] have proposed a CSA circuit for the first time in 1987. They aimed to design a preamplifier suitable for avalanche photodiode-based scintillation detectors in the range of 5-20 $\mathrm{MHz}$ frequency. In this proposal, they used Discrete MOSFET technology and proved its outperforming characteristics over BJT, JFET, and GaAs MESFET in terms of cost, ENC, and power consumption. BJTs and JFETs were commonly used for detectors at that time. They have enhanced a preamplifier circuit with $300 \mathrm{~mW}$ power consumption for each channel. They implemented a dual-channel preamplifier resulting in a total of $600 \mathrm{~mW}$ power consumption. They designed a suitable circuit for large-scale high-density applications and this was a fair good result at that time but not comparable to what it is found recently.

Binkley et al. [4] have introduced a transimpedance preamplifier circuit using Complementary FET technology for the first time. They used $2 \mathrm{~m} \mathrm{CMOS}$ and implemented it for APD applications. In their work, they proved that the ENC in the transimpedance preamplifier is much smaller than in CSA, where the measured $\mathrm{ENC}$ was 560 electrons-rms at peaking time $=68 \mathrm{n}$ (in our work ENC equals 43). The presented circuit in [4] recorded a huge reduction in power dissipation compared to [6] with estimated power dissipation equaled 38 $\mathrm{mW}$. The problem in such a transimpedence preamplifier is the high $W / L$ ratio which in their design was 1200. This results in larger preamplifier circuit physical dimensions; thus this deficit increases the parasitic capacitances and maximizes the external interference since the preamplifier is not close enough to the detector.

Paulus et al. [3] presented a modified cascode CSA circuit and compared it with a previous simple design folded cascode circuit. The simple cascode circuits require a high $W / L$ FET to reduce the Miller effect on it - especially at the input FET - which leads to a decrement in the input capacitance of the preamplifier. However, a large $W / L$ ratio will lead to undesired chip area size as mentioned above. Also, cascode topology produces a low open loop gain. Therefore, they introduced their modified $2 \mathrm{~m}$ CMOS cascode design, which was 50 times greater in open loop gain than that of the simple cascode. They reduced the $W / L$ ratio from 1200 in the simple cascode topology to 100 , which will affect significantly the chip area. The only exception was the input FET which was PMOS with $7500 \mathrm{~m} / 2 \mathrm{~m}$ shape factor to set the amplifier input capacitance to approximately $10 \mathrm{pF}$. In this work, the authors succeeded to reduce the ENC of the detector circuit but this led to an increment in power dissipation to $50 \mathrm{~mW}$ compared to $38 \mathrm{~mW}$ in [3]. This flaw affects Thick-film fabrication disallowing high density detector arrays to be implemented and reducing image resolution.

Binkley et al. [2] had returned to the regular simple cascode CSA topology but this time with $800 \mathrm{~nm}$ CMOS technology. The reduction of channel length to such value has significantly reduced power dissipation compared to [4]. In their work, they concentrated on power consumption at the expense of chip area which will affect in consequence high density detector arrays implementation and image resolution as mentioned earlier. Binkley et al. [2] also used large $W / L$ CMOS values, ranging from 1200/0.8 m shape factor to $4800 / 0.8 \mathrm{~m}$. They used the regulated cascode preamplifier architecture. It contains 1-pF feedback capacitance in parallel with l-M $\Omega$ feedback resistor (implemented by a MOSFET in the deep-ohmic region); this feedback was used to minimize the cascode input resistance while simultaneously maximizing the cascode output resistance which will increase dc loop gain. They replaced the input PMOS used in their previous work [4] with (NMOS. The change resulted in lower input white-noise voltage since n-type metal-oxide-semiconductor (NMOS) has higher transconductance. Their proposed circuit is optimal for wideband applications.

Pratte et al. [1] introduced CSA using 180 CMOS technology. They really introduced a circuit model that reduced power consumption to less than $10 \%$ compared to work in [2]. They proposed 2 circuit models. The power dissipated in the first design was $5 \mathrm{~mW}$, and the second design was only $1 \mathrm{~mW}$ that led to an increase in the number of stacked detectors on a single chip. But in their design, they did not mention the value of $W / L$ 
ratio they had used in order to judge their design efficiency. The second issue is that the resulted ENC was very large; it recorded 1187 Electrons-rms at the peaking time compared to our 43 electrons-rms value. This reduces image quality, especially for high resolution imaging applications.

Ratti et al. [7] have introduced CSA circuit with $65 \mathrm{~nm}$ technology. They aimed to use this preamplifier in a circuit used as a time over threshold counter (TOT counter). In their research, they built a circuit based on folded cascode architecture with a regulated cascode load to boost impedance seen at the amplifier output. They used NMOS at the circuit input. A huge reduction in chip area was achieved. Many thanks to the reduction of MOSFET channel length. An equivalent noise charge of 120 e-rms. The power dissipation of the cascode CSA was $3.6 \mu \mathrm{W}$, which is slightly less than our $4 \mu \mathrm{W}$ power dissipation and that is because they used the CSA in a $1.2 \mathrm{~V}$ power plane but we use $1.8 \mathrm{~V}$. Using such a lower voltage value will be at the expense of the gain of the CSA.

To sum up, many research works have been introduced on CSA. We discussed the pros and cons of some recent works. Most of them made their designs with different topologies and had many differences, but all agreed on having CMOS channel length reduction. CMOS regime is the keyword of reducing power dissipation and reduction of ENC and this is what our paper depends on.

\section{PRELIMINARIES}

When designing a low noise charge amplifier, several design considerations should be taken into account: Thermal noise capacitive match, W/L ratio, and other parameters that we will state later in this paper [1], [3]. So, in this section, the models of ENC and Transconductance for a charge sensitive preamplifier are explained showing their controlling parameters.

\subsection{Equivalent noise charge}

The equivalent noise charge (ENC) is the traditional measurement term for describing the resolution of charge sensitive front-end electronics. The ENC corresponds to the charge that must be delivered to the front-end in order to have a unity signal-to-noise ratio [1], [4], [6] and it is measured in rms electrons. In (1) indicates the classical ENC model for a charge sensitive preamplifier [1]:

$$
E N C^{2}=\left(C_{d e t}+C_{g s}\right)^{2} \cdot\left(\frac{4 K T \gamma}{g_{m}}+\frac{K_{f}}{C_{g s}}\right)
$$

The first term i.e. $\left(C_{d e t}+C_{g s}\right)$ is due to the input MOSFET thermal noise, and the second term $\left(4 K T \gamma / g_{m}+K_{f} / C_{g s}\right)$ is the input MOSFET $1 / f$ noise. $C_{d e t}$ is the detector capacitance and $C_{g s}$ is the MOSFET gate to source capacitance, $g_{m}$ is transconductance of source input MOSFET. $K$ is the Boltzmann's constant, $K_{f}$ is flicker noise coefficient of the MOSFET, $T$ is temperature in kelvin, $\gamma$ is the coefficient of thermal noise for the input MOSFET. And we should keep in mind that capacitance matching between $C_{d e t}$ and $C_{g s}$ is needed. Figure 2 shows parasitic capacitances in NMOS and its parasitic capacitances representation on MOS geometry side by side with detector capacitances. As we will show later in the proposed circuit, the input MOSFET is NMOS. NMOS has a higher transconductance when comparing with PMOS [8]. This will decrease ENC and reduce the noise of the received signal.

There are many variables that contribute to the ENC of a MOSFET that are technology-dependent and user-defined. A designer usually has only control of the $W / L$ ratio of the transistor and therefore it is limited in the noise optimization process [22], [23]. The only parameters in (1) that the designer has control over is $C_{g s}$ an optimum value for $C_{g s}$ must exist since increasing or decreasing $C_{g s}$ can mean large changes in the ENC. Later we will show Equations that define $C_{g s}$ in terms of $W$ and $L$ in order to visualize how a change in $W$ and $L$ will affect each term [24], [25]. The noise of any MOSFET has 3 types: the white series noise, the white parallel noise, and the frequency noise. Figure 3 shows noise and capacitances models for CSA noise and capacitance are key parameters for CSA which gives an indication of how much this CSA is good when a certain technology is chosen, the only way to control the behavior of a component in the circuit is by changing the size of a component which we will do later. 


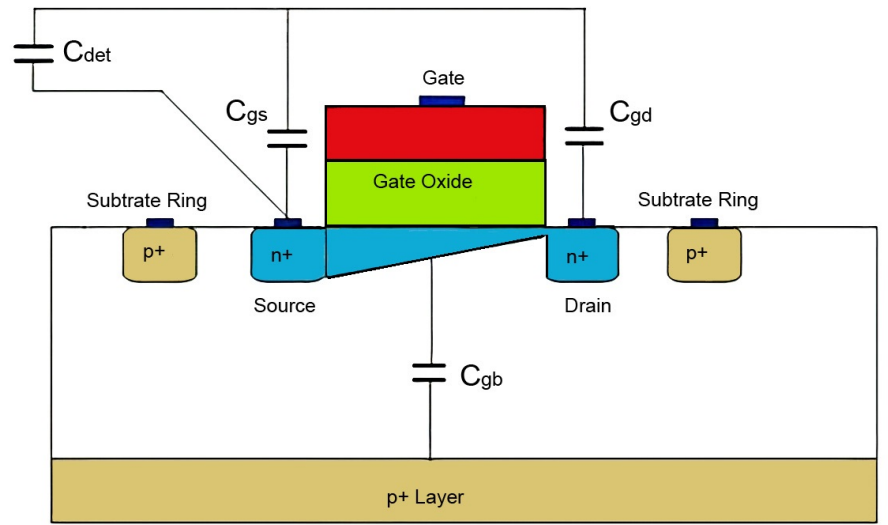

Figure 2. MOSFET overview with parasitic capacitances

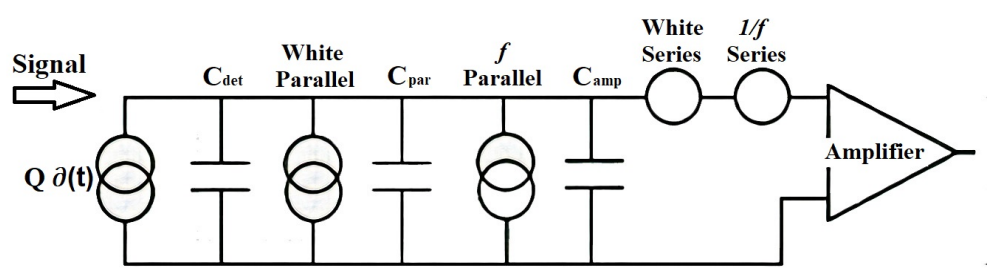

Figure 3. Noise and capacitance model for CSA

Now the general formula for ENC for any MOSFET is (2):

$$
E N C=\sqrt{E N C_{s}^{2}+E N C_{p}^{2}+E N C_{f}^{2}}
$$

where $E N C_{s}$ is measurement for series noise and $E N C_{p}$ is measurement for parallel noise and $E N C_{f}$ is measurement for frequency noise [1]:

$$
\begin{gathered}
E N C_{f}^{2}=C_{i n}^{2} \frac{K_{F N}}{C_{o x} W L} \pi A_{f} \\
E N C_{p}^{2}=\frac{2 K T A_{p} \tau_{p}}{R_{e q}} \\
E N C_{s}^{2}=\frac{2 K T A_{s} C_{i n}^{2}}{\tau_{p}}\left(\frac{\gamma}{g_{m n}}+R_{p}\right)
\end{gathered}
$$

where $A_{f, p, s}$ are the form factors of input the shaping function, $C_{i n}$ is total capacitance at the CSA input, $T_{P}$ is the shaping function peaking time, $K_{F N}$ is the NMOS flicker noise coefficient, $R_{P}$ is the parasitic resistance in series with each transistor electrode, $C_{o x}$ is the gate oxide capacitance per unit area, $W$ is the width and $L$ is the length of the input NMOS, and $R_{E Q}$ is the equivalent resistance of the rest reading out system [1], [7].

\subsection{Transconductance}

One of the important parameters of a FET is the transconductance $g_{m}$. The formula for the $g_{m}$ is:

$$
g_{m}=\sqrt{2 \cdot \mu_{e f f} \cdot C_{o x} \cdot \frac{W}{L} \cdot I_{D S}}
$$

where $I_{D S}$ is the drain to source current, and $\mu_{e f f}$ is the charge-carrier effective mobility [20], [21].

For strong inversion mode: 


$$
C_{g s}=\frac{2}{3} C_{o x} \cdot W L
$$

Now, solving the (7) for $C_{o x}$ to find the relation between $L$ and $g_{m}$ produces the (8).

$$
g_{m}=\sqrt{\frac{3 \mu_{N} \cdot C_{g s} \cdot I_{D}}{L^{2}}}
$$

If we look for the (8), we can figure out that transconductance is inversely proportional to the channel length. So reduction of technology means larger transconductance and conductance are achieved.

\section{SCALING}

The concept of scaling has evolved with the revolution of sub $100 \mathrm{~nm}$ technology. It is worth indicating that by scaling, we mean that the effective channel length is reduced by a scale of $S$. However, scaling can be challenging because when we reduce channel length by a scale $S$ (for ; $100 \mathrm{~nm}$ technology) some parameters have to be changed to preserve currents and voltage relations in MOSFET, as described later. In addition, some nonlinearities will appear and should be taken into consideration [26].

In constant-voltage scaling, all dimension of the MOSFET are reduced by a factor of $S$ [23], [24]. The power supply voltage will not be changed. The doping densities $N_{A}$ and $N_{D}$ must be increased by a factor of $S^{2}$ in order to preserve the charge-field, as listed in Table 1 [25]. When applying such scaling, the MOSFET parameters will be changed as seen in Table 2 [25].

Table 1. Constant voltage scaling

\begin{tabular}{lll}
\hline Quantity & Before scaling & After scaling \\
\hline Dimensions & $W, L, t_{o x}, x_{j}$ & Reduced by $S$ \\
Voltages & $V_{D D}, V_{T}$ & Remain unchanged \\
Doping densities & $N_{A}, N_{D}$ & Increased by $S^{2}$ \\
\hline
\end{tabular}

Table 2. MOSFET parameters before and after scaling

\begin{tabular}{lll}
\hline Quantity & Before scaling & After scaling \\
\hline Channel width & $\mathrm{W}$ & $W^{\prime}=W / S$ \\
Channel length & $\mathrm{L}$ & $L^{\prime}=L / S$ \\
Gate oxide thickness & $t_{O x}$ & $t_{o x}^{\prime}=t_{o x} / S$ \\
Junction depth & $x_{i}$ & $x_{j}=x_{i} / S$ \\
Oxide capacitance & $C_{o x}$ & $C_{o x}^{\prime}=S \cdot C_{o x}$ \\
Drain current & $I_{D}$ & $I_{D}^{\prime}=S \cdot I_{D}$ \\
Threshold voltage & $V_{T 0}$ & $V_{T 0}^{\prime}=V_{T 0}$ \\
Power supply voltage & $V_{D D}$ & $V_{D D}^{\prime}=V_{D D}$ \\
Doping densities & $N_{A} \& N_{D}$ & $N_{A}^{\prime}=S^{2} \cdot N_{A} \& N_{D}^{\prime}=S^{2} \cdot N_{D}$ \\
\hline
\end{tabular}

Now, we will use the scaling concept described and apply it on a $180 \mathrm{~nm}$ predesigned CSA [1], [10], [14], and replace all transistors with $45 \mathrm{~nm}$ transistors. Here, we are talking about scaling by $\mathrm{S}=180 / 45=4$. Thus, we will adjust the equations of the preamplifier by considering scaling effects. And we will prove that using $45 \mathrm{~nm}$ technology will enhance the gain, the response time, ENC, and the power consumption as well.

\section{ANALYSIS}

Figure 4 shows a circuit of the CSA that we will apply our analysis and modifications on [1], [3], [4]. This preamplifier is based on modified telescopic cascode topology. The advantage of this topology comes on low power dissipation, with mid-range voltage gain, and lower noise. The Input of the preamplifier is at Q1 which is typically an NMOS. NMOS provides higher transconductance over PMOS which results in minimizing ENC. Transistor Q2 is used as the main current source to produce the desired transconductance in the input transistor [1], [4]. Q7 is used as a feedback resistor as it works in the deep ohmic region. Q7 is in parallel with the feedback capacitor $C_{F}$ which is necessary to let the CSA work in integration mode [4]. All MOSFETs have $W / L=100$ for design symmetry. $C_{F}$ and $C_{c}$ both are set to $10 \mathrm{fF}$. 


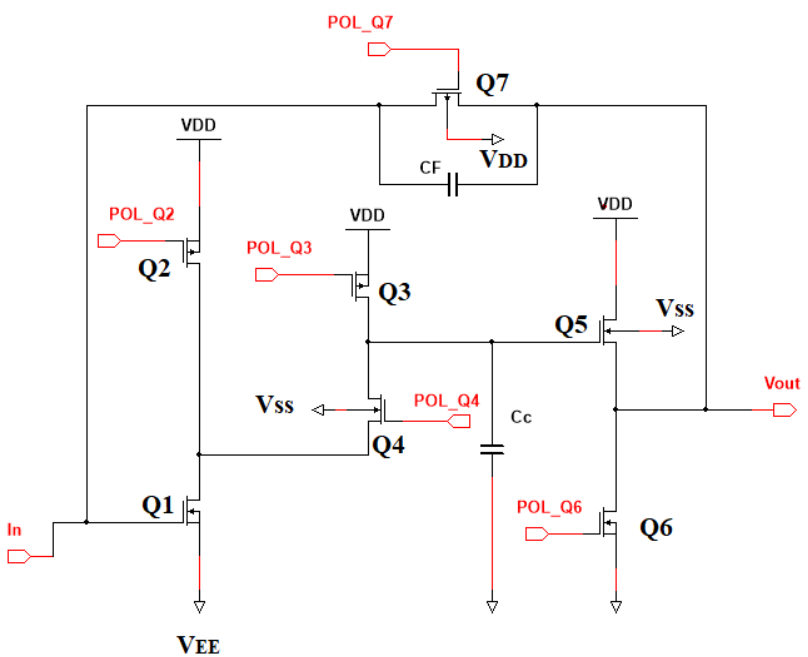

Figure 4. Main CSA circuit

Back to (6), we can note that $g_{m}$ is controlled by oxide capacitance $C_{o x}$. Also, $g_{m}$ is determined by the width of MOSFET and the channel length which in this case equals to $45 \mathrm{~nm}$. Now by considering the scaling factor $S, g_{m}$ will be scaled by $S$ and this will be useful and leads to a more efficient design.

Now substituting $L=L / S, W=W / S, C_{o x}=S . C_{o x}, I_{D}=S \cdot I_{D}$ in in (1) and (8) yields to $C_{g s}=C_{g s} / S$

$$
\begin{aligned}
& g_{m}^{\prime}=\sqrt{2 \cdot \mu_{e f f} \cdot S \cdot C_{o x} \frac{W / S}{L / S} S \cdot I_{D S}} \\
& g_{m}^{\prime}=\sqrt{2 \cdot \mu_{e f f} \cdot S^{2} \cdot C_{o x} \frac{W}{L} \cdot I_{D S}} \\
& g_{m}^{\prime}=S \sqrt{2 \cdot \mu_{e f f} \cdot C_{o x} \frac{W}{L} \cdot I_{D S}} \\
& g_{m}^{\prime}=S \cdot g_{m}
\end{aligned}
$$

Also,

$$
\begin{aligned}
& g_{m}^{\prime}=\sqrt{\frac{3 \mu_{N}\left(C_{g s} / S\right) \cdot S \cdot I_{D}}{(L / S)^{2}}} \\
& g_{m}^{\prime}=\sqrt{\frac{3 S^{2} \mu_{N} C_{g s} I_{D}}{L^{2}}}
\end{aligned}
$$

This leads also to (9). This proofs that current conductance (charges conductance) through the MOSFET channel length will be increased by factor $S$, consequently decrementing the signal distortion transmitted through MOSFET. Now substituting in (11):

$$
\begin{aligned}
E N C^{2} & =\left(\frac{C_{d e t}+C_{g s}}{S}\right)^{2}\left(\frac{4 K T \gamma}{S g_{m}}+\frac{K_{f}}{C_{g s} / S}\right) \\
& =\frac{\left(C_{d e t}+C_{g s}\right)^{2}}{S^{3}} \cdot \frac{4 K T \gamma}{g_{m}}+\frac{\left(C_{d t}+C_{g s}\right)^{2}}{S} \cdot \frac{K_{f}}{C_{g s}}
\end{aligned}
$$


Note that mathematically the new $E N C^{2}$ is reduced by $S^{3}$ for the first term and by $S$ for the second term, which really makes a great enhancement on detection accuracy over 180-Nano detectors [1]. We will see in the next section the simulation results that will assure our enhancement shown in calculations.

\section{SIMULATION RESULTS}

Simulation of our proposed enhanced design was conducted by using PSpice circuit simulator and the results was extracted to Matlab to draw graphs. Simulation results showed that ENC and signal-to-noise ratios have been more stable and have minimum distortions.

\subsection{Simulation parameters}

In our simulation, we used $W / L$ ratio equal to 100 . The size of the input device was optimized for the derivative of a third-order semi-Gaussian shaping function [1], [2], [12], [15]. Bipolar $\mathrm{CR}^{2}-\mathrm{RC}^{2}$ shaper was used with a peaking time of $70 \mathrm{~ns}$ and $1.8 \mathrm{-V}$ power and ground plane. The following Table 3 presents CR-RC, $\mathrm{CR}^{2}-\mathrm{RC}^{2}$ and the derivative of a $3 \mathrm{rd}$ order semi-Gaussian shaping function for $70 \mathrm{~ns}$ peaking time [1].

Table 3. Shaping form factors

\begin{tabular}{llll}
\hline Form factors & CR-RC & CR $^{2}-\mathrm{RC}^{2}$ & Semi-Gaussian \\
\hline$A_{f}$ & 1.18 & 1.55 & 1.61 \\
$A_{S}$ & 1.85 & 2.27 & 2.15 \\
$A_{P}$ & 1.85 & 1.45 & 2.10 \\
\hline
\end{tabular}

Detector module has a capacitance of $50 \mathrm{fF}$ and biased at $350 \mathrm{~V}$ with a Gain that is set to 55. Feedback capacitance $C_{F}$ was set to $10 \mathrm{fF}$. An input signal is injected in the circuit on the form of a test pulse applied to a characterized $15 \mathrm{fF}$ capacitor for the main circuit.

\subsection{Voltage transfer characteristics (Gain)}

Simulation results have shown that our proposed circuit has deduced a gain of $3.1 \mathrm{mV} / \mathrm{fC}$, with a maximum output swing of $100 \mathrm{mV}$, as seen in Figure 5. An input signal approximately is injected in the circuit on the form of a test pulse applied to a characterized $15 \mathrm{fF}$ capacitor for the main circuit. In obtaining this result, the input capacitance was set to $50 \mathrm{fF}$.

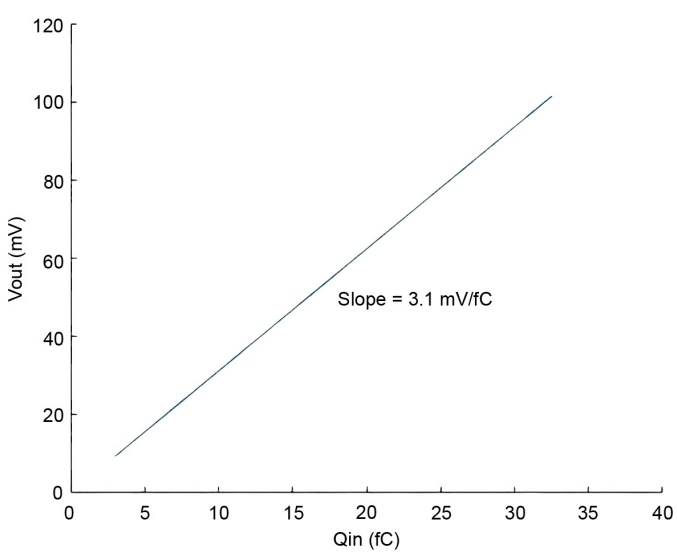

Figure 5. Gain of CSA circuit

\subsection{ENC measurement}

Figure 6 shows ENC for the peaking time range from 60 to $200 \mathrm{~ns}$. ENC is measured as a function of different input capacitances. Simulation results have shown that ENC was reduced by more than $96 \%$ using 45 $\mathrm{nm}$ technology compared to $180 \mathrm{~nm}$ technology [1]. Figure 7 shows the $\mathrm{ENC}$ with $\mathrm{ENC}_{S, P, 1 / f}$ for peaking time from $10 \mathrm{~ns}$ to $10 \mathrm{~s}$ at input capacitance $=20 \mathrm{fF}$. The resulting ENC showed minimum value of 43 electrons-rms at peaking time $=77 \mathrm{~ns}$. This result shows a great enhancement over $180 \mathrm{~nm}$ technology that recorded 1117 electrons-rms at $100 \mathrm{~ns}[1]$. 


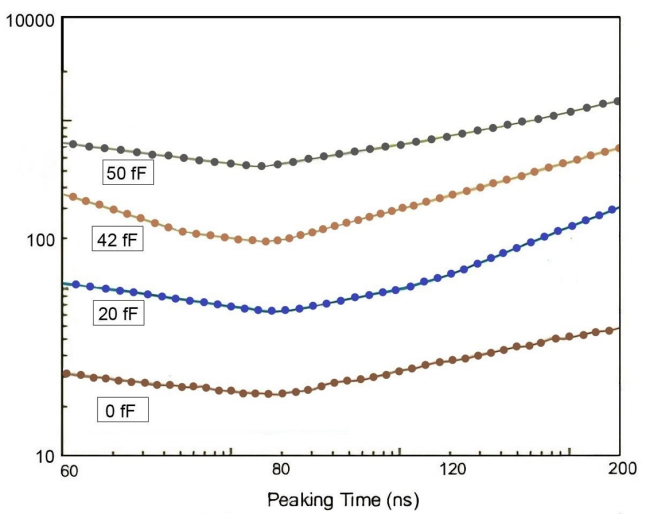

Figure 6. ENC vs peaking time for different input capacitances

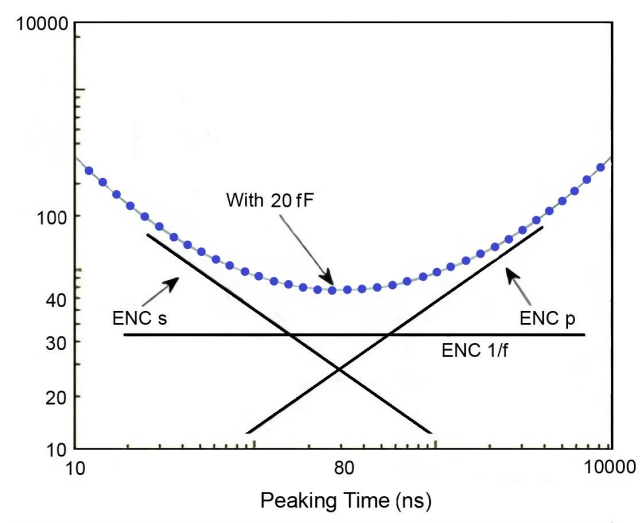

Figure 7. ENC vs peaking time

\subsection{Power dissipation measurement}

Figure 8 shows the power consumed by three different technologies for different measured ENC values. The figure clearly shows that when reducing the channel length, power dissipation is noticeably reduced by a good value.

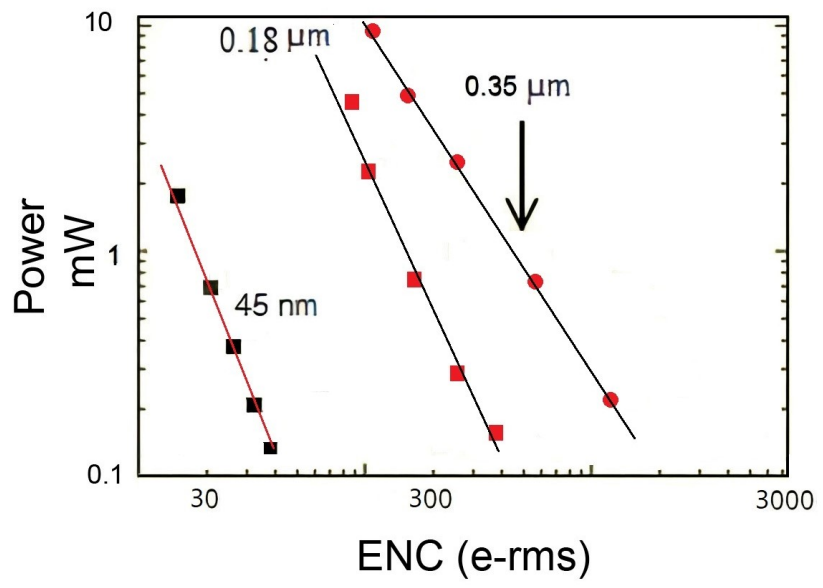

Figure 8. ENC VS power dissipation

\subsection{Comparison with other previous works}

Table 4 shows a comparison of our proposed scheme with other previous designs using main CSA different parameters. The common thing among them that all were developed for LSO/APD for PET systems. Also, all were implemented using CMOS technology except the first were developed using discrete MOSFET, not complementary. The comparison is represented in the shape of a historic timeline to show advances made with respect to time.

Table 4. Comparisons with different technologies

\begin{tabular}{|c|c|c|c|c|c|c|c|}
\hline CSA Parameters & $\begin{array}{l}\text { Schmitt } \\
\text { et al. [6] }\end{array}$ & $\begin{array}{l}\text { Binkley } \\
\text { et al. [4] }\end{array}$ & $\begin{array}{l}\text { Paulus } \\
\text { et al. [3] }\end{array}$ & $\begin{array}{l}\text { Binkley } \\
\text { et al. [2] }\end{array}$ & $\begin{array}{l}\text { Pratte } \\
\text { et al. [1] }\end{array}$ & $\begin{array}{l}\text { Ratti } \\
\text { et al. [7] }\end{array}$ & Our work \\
\hline \multirow[t]{2}{*}{ Used echnology } & Discrete & $2 \mu \mathrm{m}$ & $2 \mu \mathrm{m}$ & $800 \mathrm{~nm}$ & $180 \mathrm{~nm}$ & $65 \mathrm{~nm}$ & $45 \mathrm{~nm}$ \\
\hline & MOSFET & CMOS & CMOS & CMOS & CMOS & CMOS & CMOS \\
\hline $\mathrm{e}_{n}\left(\mathrm{nV} / \mathrm{Hz}^{1 / 2}\right)$ & 0.9 & 1.6 & 1.1 & 0.65 & 0.57 & N/A & 0.41 \\
\hline Current (mA) & 20 & 5 & 10 & 2 & 0.6 & 0.003 & 0.003 \\
\hline Time resolution (FWHM) & $0.9 \mathrm{~ns}$ & $9.2 \mathrm{~ns}$ & $1.57 \mathrm{~ns}$ & $1.15 \mathrm{~ns}$ & $2.49 \mathrm{~ns}$ & N/A & $82 \mathrm{ps}$ \\
\hline Rise time (using $0 \mathrm{pF}$ ) (ns) & 5 & 22 & 7 & 6 & 9 & 17 & 5 \\
\hline Power (mW) & 300 & 38 & 50 & 10 & 1 & 0.0036 & 0.004 \\
\hline
\end{tabular}


- Technology used all CSA-based PET applications use CMOS as mentioned before and we notice that as time passes MOSFETS are minimizing their channel length. Our proposed circuit uses the $45 \mathrm{~nm}$ MOSFET, which is considered one of the minimal channel length currently used in such circuits.

- The noise spectral density is preserved between 0.41 and 1.6 in all of the above designs. It is known that the ENC is directly proportional to the spectral noise density $e_{n}$. In our work, this parameter is reduced by $25 \%$ compared to $180 \mathrm{~nm}$ technology [1]. This is due to higher transconductance in shorter channel MOSFETS.

- The feeding current is reduced with recent designs. Our 3 A circuit is very suitable for high dense applications.

- Time resolution full width at half maximum (FWHM) was reduced massively by more than 95\% compared to [1] which is breaking through a constant fraction discriminator and a CR-RC Timing Filter Amplifier connected directly to CSA output to measure time resolution.

- Rise time is reduced by $45 \%$ compared to [1] which results in a faster responsive amplifier circuit.

- Power consumption is decreased due to the reduced channel length and that's why all most recent works are competing in shrinking CMOS used in CSA design. Our power reduction breakthrough will pave the road for higher dense - higher-resolution medical imaging applications.

\section{CONCLUSION AND FUTURE WORK}

This paper has introduced an efficient design of a low noise charge amplifier using $45 \mathrm{~nm}$ technology, which is made for the first time in such circuits. The proposed scaling technology with the amplifier design improves the efficiency in terms of limiting noise effect while maintaining amplification value. This will enhance the quality of received images in terms of resolution and accuracy in many PET and wireless applications. Simulation results proved that direction toward deep submicron CMOS circuit by shrinking transistors size is a powerful trend that leads to reduce noise, power consumption, ENC, noise spectral density, and time resolution. We have made our comparison mainly on $180 \mathrm{~nm}$ technology and the results show a great enhancement in ENC value by more than $95 \%$ and lowering power consumption by more than $95 \%$. In addition, our comparisons consider other older technologies such as $350 \mathrm{~nm}$, and $800 \mathrm{~nm}$. Future work would concentrate on optimizing response time and frequency response. This would be a concrete step towards more interesting research lines. Moreover, our analysis on operating Q1 MOSFET assumed strong inversion operation mode, while it really works in a moderate-to-strong inversion region resulting in shifting the minimum ENC value at 70 ns peaking time to $77 \mathrm{~ns}$. Future studies should take this into consideration in order to enhance results and their accuracy. In addition, researchers may test our proposed design's performance with Monte-Carlo simulation for the ENC variation for extra analysis.

\section{ACKNOWLEDGEMENT}

This research work was funded and supported by Hashemite University - Jordan, and this research was undertaken, in part, thanks to funding-from the Canada Excellence Research Chairs Program". The funding source is Tri-Agency Institutional Program Secretariat.

\section{REFERENCES}

[1] S. Robert et al., "Design and performance of 0.18-/spl mu/m CMOS charge preamplifiers for APD-based PET scanners," 2003 IEEE Nuclear Science Symposium. Conference Record (IEEE Cat. No.03CH37515), 2003, pp. 1242 1246, vol.2, doi: 10.1109/NSSMIC.2003.1351917.

[2] D. M. Binkley, B. S. Puckett, M. E. Casey, R. Lecomte and A. Saoudi, "A power-efficient, low-noise, wideband, integrated CMOS preamplifier for LSO/APD PET systems," in IEEE Transactions on Nuclear Science, vol. 47, no. 3, pp. 810-817, June 2000, doi: 10.1109/23.856522.

[3] M. J. Paulus, J. M. Rochelle, M. S. Andreaco and D. M. Binkley, "A low-noise, wide-band CMOS charge-sensitive preamplifier for use with APD/LSO PET detectors," 1995 IEEE Nuclear Science Symposium and Medical Imaging Conference Record, 1995, pp. 330-334, vol.1, doi: 10.1109/NSSMIC.1995.504238.

[4] D. M. Binkley, J. M. Rochelle, M. J. Paulus, and M. E. Casey, "A low-noise, wideband, integrated CMOS transimpedance preamplifier for photodiode applications," IEEE Transactions on Nuclear Science, vol. 39, no. 4, pp. 747-752, 1992.

[5] F. Javaid, M. Rashid, and S. Khan, "A configurable high resolution digital pixel readout integrated cir- 
cuit with on-chip image processing," Computers \& Electrical Engineering, vol. 86, p. 106720, 2020, doi: 10.1016/j.compeleceng.2020.106720.

[6] D. Schmitt et al., "Ultra-Low Noise Charge Sensitive Preamplifier for Scintillation Detection with Avalanche Photodiodes in PET Applications," in IEEE Transactions on Nuclear Science, vol. 34, no. 1, pp. 91-96, Feb. 1987, doi: 10.1109/TNS.1987.4337308.

[7] L. Ratti, F. De Canio, L. Gaioni, M. Manghisoni, V. Re and G. Traversi, "A Front-End Channel in $65 \mathrm{~nm}$ CMOS for Pixel Detectors at the HL-LHC Experiment Upgrades," in IEEE Transactions on Nuclear Science, vol. 64, no. 2, pp. 789-799, Feb. 2017, doi: 10.1109/TNS.2016.2646908.

[8] A. Itradat and A. Bqerat, "A Hybrid Scheme for Minimizing Leakage Current in CMOS-Based Architectures by Employing Multiple Supply Voltages and Power Gating Techniques,” 2018 2nd European Conference on Electrical Engineering and Computer Science (EECS), 2018, pp. 100-104, doi: 10.1109/EECS.2018.00027.

[9] Y. Yu et al., "Analysis and Design of Inductorless Wideband Low-Noise Amplifier With Noise Cancellation Technique," in IEEE Access, vol. 5, pp. 9389-9397, 2017, doi: 10.1109/ACCESS.2017.2692765.

[10] Y. Xin, X. Zhao, B. Wen, L. Dong and X. Lv, "A High Current Efficiency Two-Stage Amplifier With Inner Feedforward Path Compensation Technique," in IEEE Access, vol. 8, pp. 22664-22671, 2020, doi: 10.1109/ACCESS.2020.2967870.

[11] Y. Liu et al., "Implementation of a Low Noise Amplifier With Self-Recovery Capability," in IEEE Access, vol. 7, pp. 43076-43083, 2019, doi: 10.1109/ACCESS.2019.2907524.

[12] Z. Zhou, "A Front-End Amplifier with Current Compensation Feedback Input Impedance Booster for Neural Signal Applications," in IEEE Access, vol. 8, pp. 178055-178062, 2020, doi: 10.1109/ACCESS.2020.3026178.

[13] C. Zhao, J. Liu, F. Shen, and Y. Yi, "Low power CMOS power amplifier design for RFID and the Internet of Things," Computers \& Electrical Engineering, vol. 52, pp. 157-170, 2016, doi: 10.1016/j.compeleceng.2015.06.006.

[14] F. Gao, L. Guo, H. Li, J. Liu and J. Fang, "Quantizer Design for Distributed GLRT Detection of Weak Signal in Wireless Sensor Networks," in IEEE Transactions on Wireless Communications, vol. 14, no. 4, pp. 2032-2042, April 2015, doi: 10.1109/TWC.2014.2379279.

[15] S. Hong, S. Lee, J. Lee and M. Je, "A Multi-Mode ULP Receiver Based on an Injection-Locked Oscillator for IoT Applications," in IEEE Access, vol. 8, pp. 76966-76979, 2020, doi: 10.1109/ACCESS.2020.2989192.

[16] Y. Gao, H. Ma, D. Abbott and S. F. Al-Sarawi, "PUF Sensor: Exploiting PUF Unreliability for Secure Wireless Sensing," in IEEE Transactions on Circuits and Systems I: Regular Papers, vol. 64, no. 9, pp. 2532-2543, Sept. 2017, doi: 10.1109/TCSI.2017.2695228.

[17] Jian-yu Lu and J. L. Waugaman, "Development of a linear power amplifier for high frame rate imaging system [biomedical ultrasound imaging applications]," IEEE Ultrasonics Symposium, 2004, 2004, pp. 1413-1416, Vol.2, doi: 10.1109/ULTSYM.2004.1418064.

[18] Y. Gong, H. Shan, Y. Teng, H. Zheng, G. Wang and S. Wang, "Deeply-Supervised Multi-Dose Prior Learning For Low-Dose Pet Imaging," 2020 IEEE 17th International Symposium on Biomedical Imaging Workshops (ISBI Workshops), 2020, pp. 1-4, doi: 10.1109/ISBIWorkshops50223.2020.9153450.

[19] K. Ryoo, M. Chilukuri and S. Jung, "A low power and low noise preamplifier circuit for hearing aid devices," 2016 IEEE Dallas Circuits and Systems Conference (DCAS), 2016, pp. 1-4, doi: 10.1109/DCAS.2016.7791128.

[20] Y. Su and X. Liu, "Design of a low noise low power preamplifier used for portable biomedical signal acquisition," 2016 9th International Congress on Image and Signal Processing, BioMedical Engineering and Informatics (CISP-BMEI), 2016, pp. 1742-1745, doi: 10.1109/CISP-BMEI.2016.7852998.

[21] S. Chang, S. Park and E. Yoon, "Low-Power Low-Noise Pseudo-Open-Loop Preamplifier for Neural Interfaces," in IEEE Sensors Journal, vol. 17, no. 15, pp. 4843-4852, Aug. 2017, doi: 10.1109/JSEN.2017.2717787.

[22] G. Scandurra, G. Cannatà, G. Giusi and C. Ciofi, "A differential-input, differential-output preamplifier topology for the design of ultra-low noise voltage amplifiers," 2017 International Conference on Noise and Fluctuations (ICNF), 2017, pp. 1-4, doi: 10.1109/ICNF.2017.7985947.

[23] M. Kumar, "Effects of scaling on MOS device performance," in IOSR Journal of VLSI and Signal Processing (IOSRJVSP), vol. 5, 2015, pp. 25-28, doi: 10.9790/4200-05132528.

[24] A. Verma, A. Mishra, and P. Mishra, "Scaling of dimensions and gate capacitances of MOSFET," in International Journal of Advanced Research in Computer Engineering \& Technology (IJARCET), vol. 1, no. 9, pp.272-275, 2012.

[25] G. Hiblot, Q. Rafhay, F. Boeuf and G. Ghibaudo, "Impact of short-channel effects on velocity overshoot in MOSFET," 2015 IEEE 13th International New Circuits and Systems Conference (NEWCAS), 2015, pp. 1-4, doi: 10.1109/NEWCAS.2015.7182061.

[26] A. Kumar and S. S. Singh, "Optimizing FinFET parameters for minimizing short channel effects," 2016 International Conference on Communication and Signal Processing (ICCSP), 2016, pp. 1448-1451, doi: 10.1109/ICCSP.2016.7754396. 


\section{BIOGRAPHIES OF AUTHORS}

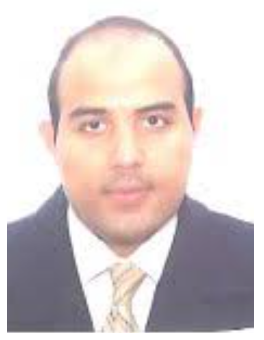

Islam T. Almalkawi (D) $5 \mathrm{SC}$ P received his Ph.D. degree in 2013 from Polytechnic University of Catalunya (UPC), the M.Sc. degree in 2008 from Carnegie Mellon University (CMU), and BSc. degree in 2004 from Hashemite University (HU). He is currently an Associate Professor in the Computer Engineering Department at Hashemite University (HU). Dr. Almalkawi worked as Research Assistance in several European Union projects during master and Ph.D. studies. His research interests include Wireless Sensor Networks, Multimedia Networks, Wireless Network Security, Image Processing, Cognitive Radio Networks, Internet of Things Networks, and Smart and Green Wireless Systems. He could be contacted via email: eslam.malkawi@ @u.edu.jo.

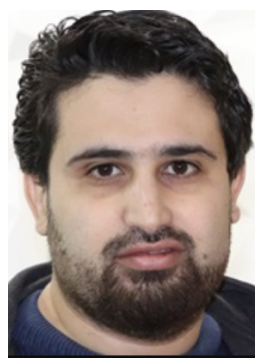

Ashraf H. Al-Bqerat $(D)$. 58 is currently a PhD candidate in Electrical and Computer Engineering at Concordia University in Canada. He has received his B.Sc. degree in Electrical and computer engineering in 2009 from Hashemite University (HU) in Jordan. Ashraf has worked at MobileCozmo initiative for SEO and e-commerce services. His research interests Include Operating systems and Memory management, Computer architecture, Semiconductors, and Nanomaterials. He could be contacted via email: ashraf.al-bqerat@mail.concordia.ca.

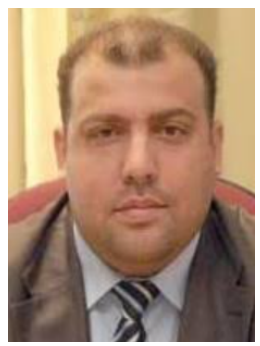

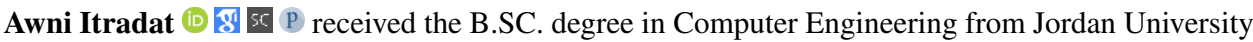
of Science and Technology, Jordan in 2000, and the Master and Ph.D. degrees in Computer Engineering from Concordia University, Montreal, Canada in 2008. He is currently a Professor in the Department of Computer Engineering and the dean of Engineering Faculty, Hashemite University. In 2009, he was appointed as the chairman of the Department of Computer Engineering at the Faculty of Engineering in Hashemite University. He has also served as a Director of the Computer Center and as the director of the ICT and E-learning Center in the Hashemite University. His research interests include Computer Architecture and Networks, Design of VLSI circuits and systems, Interconnect Modeling and Design, Reconfigurable Circuits, High-Level Synthesis of 3D- and 2D-Circuits and Systems. He could be contacted via email: itradat@hu.edu.jo.

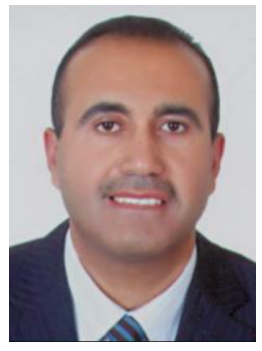

Jamal Al-Karaki (D) 80 ( 5 received the PhD degree in Electrical/Computer Engineering from the Iowa State University, USA. He has a record of progressive responsibility and authority including leadership positions as a college Dean, Dean of Academic operation, Director of IT, Co-Founder and Division Head at various institutes. He led the development some national centers as well as undergraduate program and graduate programs. He is also well versed and experienced with local and international accreditation of academic programs through CAA, NQA, and ABET standards and criteria. He published $80+$ refereed technical articles in scholarly international journals and conference proceedings. Attended/gained reputable professional certificates that includes CISSP, OSCP, ECSA, GMOB, CHFI, RHCSA, and CCNA security. He also served on the Editorial Board of some international journals and as publicity chair and technical program committee member of several International conferences and workshops. His main research interests include all aspects of computing and technology and their applications for better life. Dr. Al-Karaki is a senior member of IEEE and member of ACM and Tau Beta Pi. He could be contacted via email: jamal.alkaraki@adpoly.ac.ae. 